\title{
HaptiChem: Haptic and Visual Support in Interactions with the Microscopic World
}

\author{
Elisa Magnanelli ${ }^{1}$, Gianluca Brero ${ }^{1}$, Rosa Virginia Espinoza Garnier ${ }^{1}$, \\ Giacomo Mazzoletti ${ }^{2}$, Alessandro Maria Rizzi ${ }^{2}$, and Sara Comai ${ }^{2}$ \\ 1 Politecnico di Torino, \\ Corso Duca degli Abruzzi, 24, 10129 Torino, Italy \\ 2 Politecnico di Milano, \\ Piazza Leonardo Da Vinci, 32, 20133 Milano, Italy \\ sara.comai@polimi.it, \\ \{gianluca.brero, rosa.garnier, elisa.magnanelli, \\ giacomo.mazzoletti, alessandro.rizzi\}@asp-poli.it
}

\begin{abstract}
Haptic technologies provide physical sensations in the interaction with a computing system, by exploiting the human sense of touch and by applying forces, vibrations, or motions to the user hands or body. Considering their features, they can be a useful tool in life-science teaching, especially when molecules are involved. For this purpose, a framework composed of an haptic device and a visual interface for molecular exploration has been developed to simulate molecular and intermolecular interactions. Furthermore, this work evaluates the visual and haptic tool for molecular exploration in a didactic context, performing tests and interviews with students. The final aim is to properly develop the features of the tool, in order to make it suitable for the introduction in chemistry education. Preliminary results show positive and effective responses and learning gains from the tasks. It has also been noticed that the use of such an innovative instrument raises the interest of students in the learning process, which is one of the main benefits of the haptic device.
\end{abstract}

Keywords: Haptics, Intermolecular Interaction, Life-Science Education.

\section{Introduction}

Currently, life-science teaching makes a wide use of visual representations, especially when molecules are involved. Indeed, due to the miniscule size of molecular level, these aids represent an abstract knowledge that can be difficult for students to grasp. Visual perception is usually superior if compared to touch: first of all, it is rapid, while touch involves sensory exploration over time and space. However, visual representation does not provide an immediate method to improve comprehension of how molecules interact with each other. In this context, the haptic technology enables users to apply and feel forces, which would otherwise not be possible. Haptics can be extremely useful in several educational topics where forces play a fundamental role, like in the case of molecular interactions. 
A study by Bivall et al. 1] recognized the beneficial effect of using an haptic device in the solution of protein-ligand docking problems, while Schönborn et al. 2] demonstrated that when protein-ligand recognition tasks are accomplished using a visual-haptic support, less errors occur and that the conceptual understanding improves, with respect to the case where just the visual channel was used. Sourina et al. 3] proposed a visual haptic-based biomolecular docking system, developed for research in helix-helix docking typically occurring in drug design, and proposed its application in e-learning. In Sauer et al. 4] work, a Phantom haptic device was used to interact with atoms and to build molecules in a $3 \mathrm{D}$ virtual world populated by hydrogen, carbon, and oxygen atoms. The user could feel the strength of molecular bonding, properties of full and partiallyfull valence shells in bonding, and see how these properties affect the geometric structure of the molecule.

Given these premises, in a previous work, a virtual environment for the exploration of the space around molecules has been developed [5], and the usability of the tool has been assessed [6. After a further extension of the tool in order to include the simulation of the interaction between two molecules, the tool has been tested in order to evaluate the impact of its use in a chemistry learning context, and results are addressed in the present work.

The paper is organized into two main parts: in the first one, the framework and its Graphical User Interface (GUI) is presented. Some possible applications of the system are also mentioned. The second part outlines the tests and experiences carried out together with students, and the benefits that our project can give to the didactic field are highlighted. We conclude by presenting the current state of the project and possible future developments.

\section{The System}

Fig. 1 sketches the architecture of the main components of our system. The framework consists of a visual interface showing a 3D representation of a molecule. It is possible to set different modes of 3D molecular rendering among the ones that are typically used in chemistry teaching [7]: some are based on the geometrical structure (e.g., ball-n-stick), while others depend on the involved chemical elements (e.g., covalent or Van der Waals spacefill). The space around the molecule can be explored with the Sensable Phantom Omni haptic device [8] (Fig. 2). The haptic probe can be associated with two different kinds of objects:

- a point charge: with this mode, the user can explore the electrostatic field of the molecule [9]. The entity and the nature of the charge (positive or negative) can be set by the user;

- a molecule: in this case, the user can experience the interactions between the two molecules, as the probing molecule moves in 3D space around the fixed molecule. Both the fixed and the probing molecules can be chosen from a repository of molecules.

In order to simulate the aforementioned phenomena, two different typologies of information are needed: 
- Geometrical molecular data to determine how the atoms are located in the 3D space. This information must be provided as input, and for this purpose Protein Data Bank (PDB) files [10] are used;

- Electrostatic field data, that determines the intensity of the forces, and that is calculated from the geometrical information through the General Atomic and Molecular Electronic Structure System (GAMESS [11) whose output is elaborated by a second tool (wxMacMolPlt) and returns the molecular electrostatic potential (MEP) of the molecule. MEP values are arranged as a grid of voxels around the molecule up to a certain threshold distance in space $[9]$.

A zero MEP value is assumed for the points outside the grid. This is a realistic assumption because molecular interactions are short-range, and they rapidly decrease as the intermolecular distance increases [12]. Since the intensity of the returned forces is naturally very weak (nanoscale), forces are rescaled according to the range of force intensities the Phantom can transmit 12 . In order to solve the problems related to the discontinuous nature of the MEP grid, the force is computed as a linear interpolation in positions between the grid points 9 .

To simulate the molecule-molecule interaction, which represents a novel element of this work, the probing molecule is modeled as an aggregate of concentrated charges [13. Each charge interacts according to its position in the MEP of the fixed molecule. The resulting force is calculated as a sum of the single forces (e.g. Fig. 3 shows a water molecule with its three single forces). To reproduce the nuclear repulsion between atoms of different molecules, the maximum repulsive force that the device can generate is applied when the distance between the atoms reaches the critical limit.

The user can also manipulate the fixed molecule in the 3D space, by rotating, translating or zooming it. In the charge-molecule example in Fig. 4, the Van der Waals representation has been chosen and the surfaces of the atoms are colored according to the electrostatic field associated to these points. The color scale to be used can be set by the user through the bar shown on the right-hand side of the virtual environment. Moreover, other auxiliary visual information is shown; for example, the plot of the electrostatic field along the direction connecting the probe position and the center of the molecule (shown in the rectangle in the bottom-right-hand side of the interface). This information can be useful in the determining the direction and magnitude of the interaction, helping in the understanding of the binding mechanism. In this way, the student receives information on the bonding process through two information channels: the haptic one and the graphical one.

Tests regarding real-time performances have decreed that the molecule size that our tool can support ranges up to 264-308 atoms. This range allows the exploration of all the cases a student may face during high school/basic university courses. 


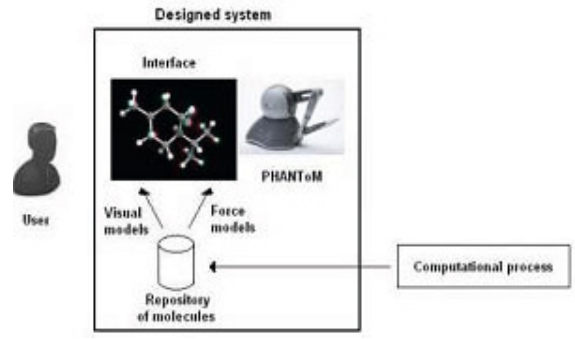

Fig. 1. Architecture of the system

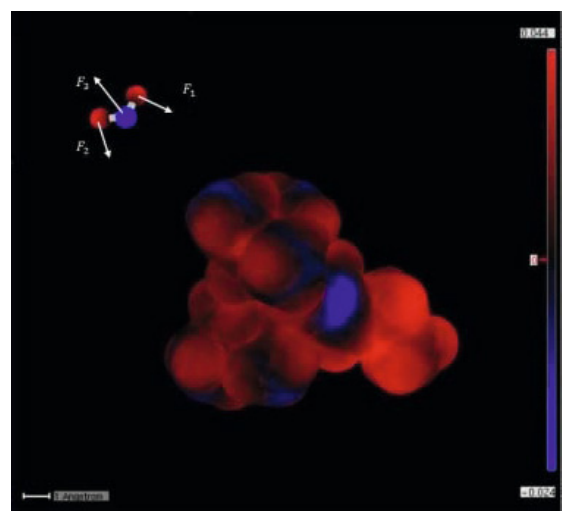

Fig. 3. Ball and stick modality: waterburane interaction

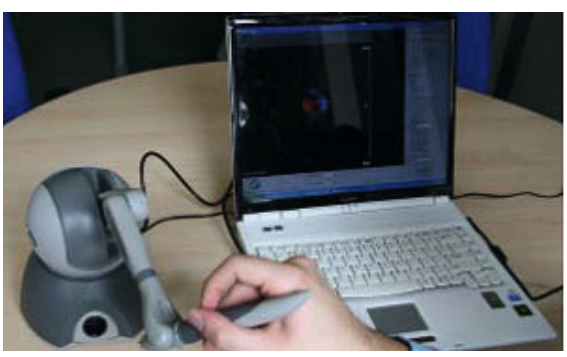

Fig. 2. HaptiChem framework

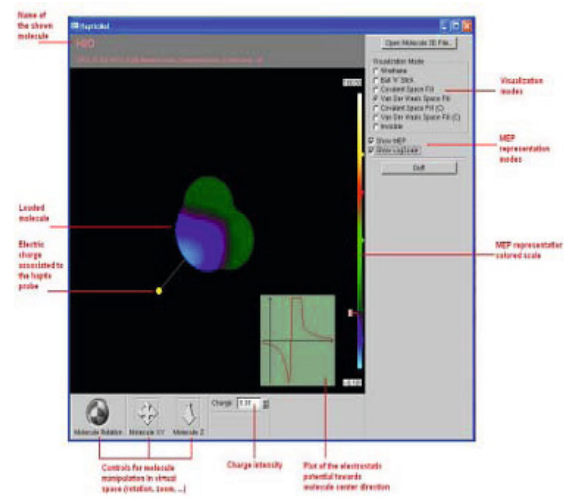

Fig. 4. Screen-shot of the tool: positive charge-water molecule interaction

\section{Possible Scenarios for Educational Activities}

Some examples of didactic activities that can be performed with the moleculecharge interaction mode have been presented in a previous work of ours $[5]$.

Therefore, we now focus on some possible examples on the molecule-molecule interaction.

- Hydrogen bonding: Because of the different electronegativity of the oxygen and the hydrogen, the molecule of water is a permanent dipole. In particular, oxygen represents the negative pole of the molecule while the hydrogens represent the positive ones [14. This feature is responsible for the hydrogen bonding between water molecules, where the partially positive hydrogen atoms are attracted by the partially negative oxygen ones of other water molecules. This phenomenon can be easily felt with the tool (Fig. 7), since the attractive force reproduced by the haptic device guides the probing molecule towards the bonding site. 
- Halogen bonding: Another intermolecular interaction that is both interesting for chemistry research activities and supported by our tool is the halogen bonding. A halogen bond $R-X \bullet \bullet Y-Z$ occurs when there is evidence of a net attractive interaction between an electrophilic region on a halogen atom $X$ belonging to a molecule or a molecular fragment $R-X$ (where $R$ can be another atom, including $X$, or a group of atoms) and a nucleophilic region of a molecule, or molecular fragment, $Y-Z$ [15. Around the halogen atoms the potential is expected to be all negative. However, some recent studies based on quantum-mechanics showed that in some molecules there is a region at the edge of the halogen atoms that has a positive potential [16]. This phenomenon can be reproduced by the tool, as in the case of the hydrogen bonding. Fig. 10 shows the interaction between an ammonia molecule $\left(\mathrm{NH}_{3}\right)$ and the trifluorine-iodio-methane $\left(\mathrm{CF}_{3} I\right)$. It can be noticed that the negative pole of the nitrogen atom of the ammonia molecule is attracted by the positive charged surface on the iodine. Since chemistry aspects are not the main topic in the present paper, we redirect the reader to our website (http://www.haptichem.com/), and we dedicate the rest of the paper to the experiences and the test performed with students.

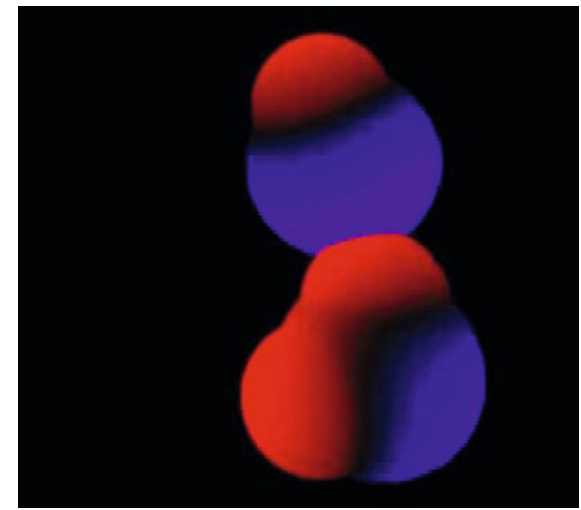

Fig. 5. Hydrogen bonding: Water-Water interaction

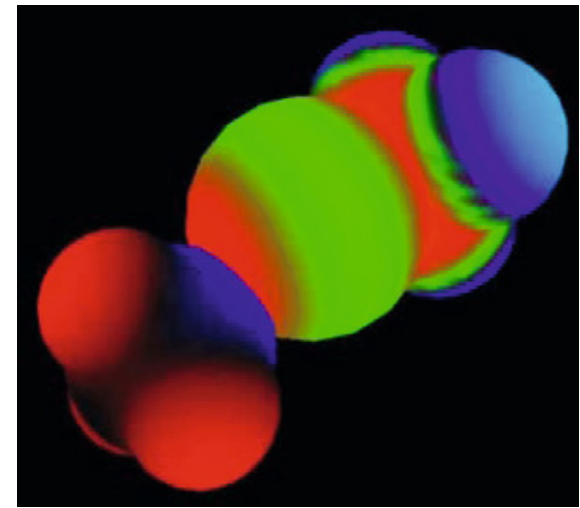

Fig. 6. Halogen bonding: AmmoniaTetraFluoroIodioMethane interaction

\section{Evaluation of the Tool}

The tool has been tested in an informal way by different kinds of users (students, teachers, researchers, but also non-experts) in different occasions (ICCEECRICE Conference in Rome1, MeetMeTonight 2012 night in Milan) and using different molecules stored in the repository. All the users who tested the tool recognized the possibility of combining the typical visualization of chemical data

1 http://www . iccecrice2012.org/ 
with the rendering of interactions between molecules provided by the haptic device. Students greatly appreciated the novelty represented by the introduction of such a tool in the current teaching activities, in order to make concepts more understandable. Teachers and researchers appreciated the improved awareness of the simulated phenomena they usually explain or deal with theoretically.

Therefore, in this work we perform a more systematic investigation with students at Politecnico di Milano, with the aim of defining the impact of the haptic device in chemistry learning. More in detail, we want to verify what the haptic technology adds to the visual rendering, when molecular interactions are simulated. The initial target population consists of engineering students (bachelor level) enrolled in a chemistry course, which is a part of the Electronic Engineering program at Politecnico di Milano. The course contents give the fundamental basis necessary to the interpretation of chemical phenomena. In particular, one of the topics of the course is organic chemistry, and the theory provides the background to determine how functional groups produce a certain electronic distribution, and how they affect molecular interactions. Therefore, after a canonical lesson given by the professor of the course, the haptic device is presented. Thereafter, a group of students takes part in the test. The test population in the study is 19 students, subdivided into two groups and accomplishing different tasks. There are 9 participants in the group A: 2 females and 7 males, in average 19,3 year old; and 10 participants in the group B: 1 female and 9 males, in average 19,2 year old.

Group A students use the haptic device for molecular exploration. The general aim is to observe how students behave in the virtual environment. Students are asked to express impressions and comments during the experience, the final purpose being the identification of strengths and weaknesses of the tool. Group $\mathrm{B}$ is asked to accomplish two tasks, and to fill in a questionnaire. The first task can be performed only using the visualization tool, while the second task is accomplished using a tool combining the visual and haptic rendering; the purpose being to highlight the improvements that the haptic device adds in the comprehension of molecular phenomena. The tasks involve the individuation of the critical points and of the parts of the molecule that take part in bindings. Molecules that are explored are Acetic Acid and Ammonia.

Two surveys are performed in the study: a survey before the test, and a final survey as a summary of the experience. The questions in the first survey are meant for statistical purposes and gather students' basic information, such as age, gender, previous knowledge on haptics, usual studying materials. Through the analysis of the two surveys and the test results, we hope to isolate the effects due to the use of the haptic device, and to highlight the learning potential of the tool.

\section{$5 \quad$ Preliminary Result Analysis}

Results from the preliminary survey show that the approach in learning chemistry is quite traditional, since all the participants indicate that during their 
studies they mainly use books (hard copy) and computer for reading lecture notes, while just two participants use a multimedia application for improving the comprehension of chemical phenomena (Fig. 7). This fact means that there is a great potential for the expansion of the technology in this field.

Afterwards, the participants are asked to provide some information about their prior knowledge, that they have to evaluate on a scale from 1 (I do not know it at all) to 5 (I know it very well). In the figures, the abscissa indicates the number of students that express a given preference. Some specifics are shown in Fig. 8, it appears clearly that their prior knowledge about the haptic technology is very limited, while the majority of the students considers its knowledge about molecular interactions intermediate.

After the preliminary survey, the two groups are involved in two different kinds of experience. The test performed by students in Group A is meant to gather the impressions that users have the first time they use the haptic device, to highlight the strengths and the weaknesses of the tool. The most significant comments that help in understanding how to improve the tool are:

- The plot of the MEP along the direction connecting the probe position and the center of the molecule aids in understanding which direction to move to find the stability points. It would be worthy to make it a default property of the interface. However, some students have troubles in the interpretation of the plot at first; the main difficulty is that the $y$-axis moves with respect to the MEP minimum as the position of the probe changes.

- Even though it is possible to rotate the fixed molecule, it would be interesting to be able to turn it while using the haptic device, in order to further exploit the attraction force.

- The feeling of repulsion is, somehow, more difficult than attraction to be perceived with the haptic device. In this context, the MEP plot gives important additional information about repulsion, and it helps in understanding when the repulsion is present.

- Only one student (out of 9 in the group) tries independently to switch from one molecular representation to another; all the others simply use the logscale mode that is already set at the beginning of their experience. A more clear and attractive way to select the $3 \mathrm{D}$ representation mode would improve the experience, since different molecular features can be understood with the different rendering modes.

- While moving in the 3D space around the molecule, several students end up with the exploring probe "behind" the molecule, and they do not menage to get back "in front" of the molecule without help. This fact is probably due to a difficulty in properly perceiving the visual representation of the depth.

We notice that, during the experience, most of the students are more focused on the haptic rendering than on the visual one. Even so, visualization is a fundamental aspect to integrate the haptic information.

Participants in group B are asked to solve two tasks initially using only the tool for the visual representation, and then using the haptic rendering as well. The two tasks involve some aspect of the molecular polarity, and the individuation 


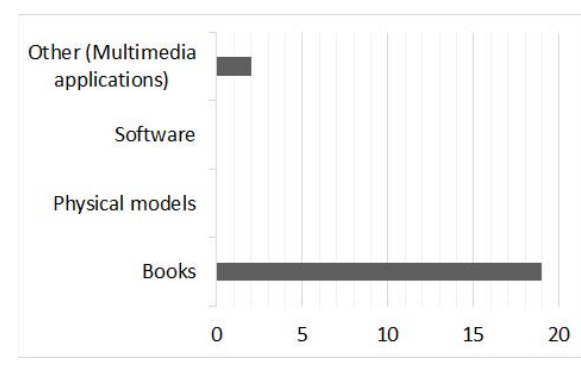

Fig. 7. Kinds of support used for studying in chemistry

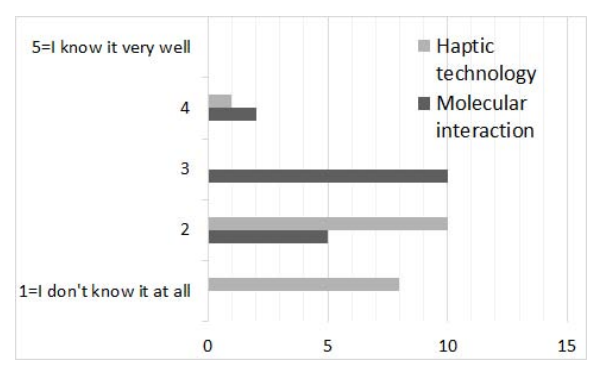

Fig. 8. Prior specific knowledge

of the points of the molecule that are critical in the bonding process. Figs. 9] and 10 show a summary of the experience. The majority of the students find it useful to integrate the visual representation with the haptic rendering, for the comprehension of molecular properties. Thanks to the haptic device, the tasks are accomplished in a more precise way, and sometimes their accomplishment is possible only when the tool is used. An improvement concerning the learning time is also noticed.

The final survey is aimed gather an overview of the opinions of the students regarding the tool, and to understand if the device is considered a valid help in basic chemistry education. The students express a very clear positive response to this new type of teaching, as more than $85 \%$ of the students included in the study consider the tool capable of generating improvements in chemistry education (Fig. 11). In general, the level of complexity of the tool is considered appropriate for the course contents and activity objectives (Fig. 12).

When students are asked to recognize the improvements in their knowledge concerning different aspects related to the basic chemistry course, mainly two aspects are highlighted: electrostatic distribution around the molecules, and the spatial understanding of the molecular structure (Fig. 13), which are the main aspects of focus of the experience. Nevertheless, some of other minor aspects that are individuated by the students can represent a valid suggestion for the application of the tool in the explanation of phenomena that have not been considered so far.

According to the students, the haptic-based tool has the potential to improve the understanding of the molecular phenomena (Fig 14), as well as to increase the spacial ability of the user in a molecular environment (Fig 15). Fig. 16] summarizes the results provided by students when asked to figure out and evaluate specific aspects of the haptic-based tool. Once more, the students recognize the awareness in the molecular perception provided by the haptic device, and most of the students agree that the learning gain due to the experience would not have been the same without the inclusion of the tool. On the other hand, they generally show to be skeptical on the usefulness of introducing sounds as an additional channel for providing information. 


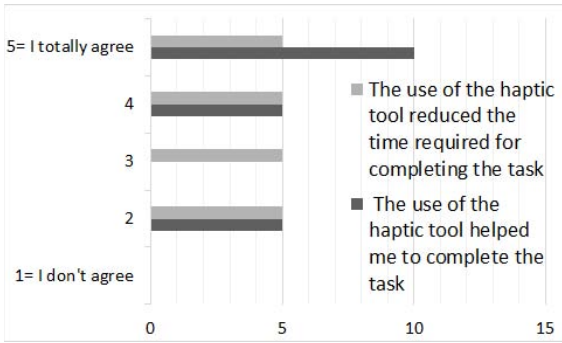

Fig. 9. Evaluation of the role of the haptic tool during the accomplishment of the task

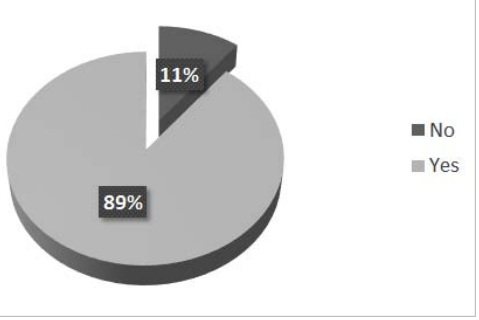

Fig. 11. Can the haptic-based tool generate improvements in chemistry education?

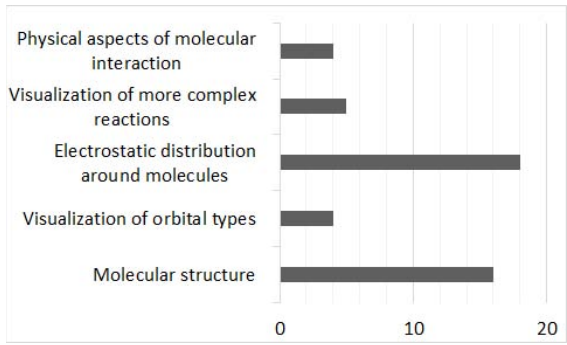

Fig. 13. Specific improvements indicated by the student

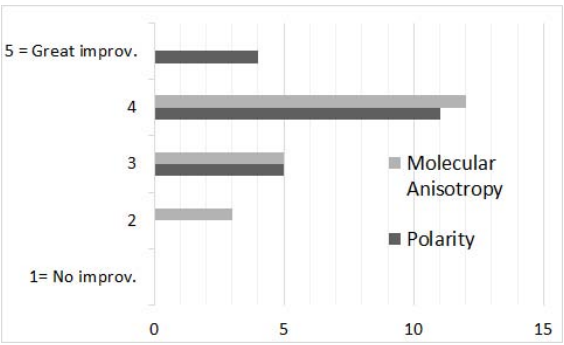

Fig. 10. Evaluation of improvements in the comprehension of molecular phenomena due to the haptic tool

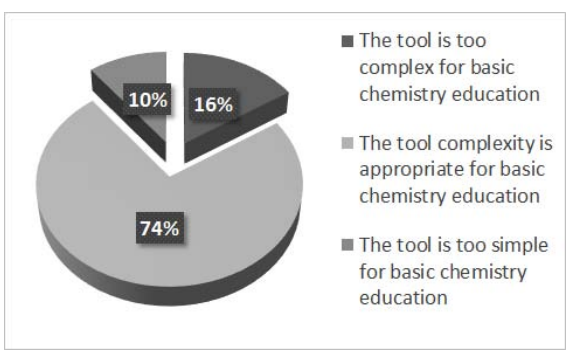

Fig. 12. Student evaluation of the tool complexity

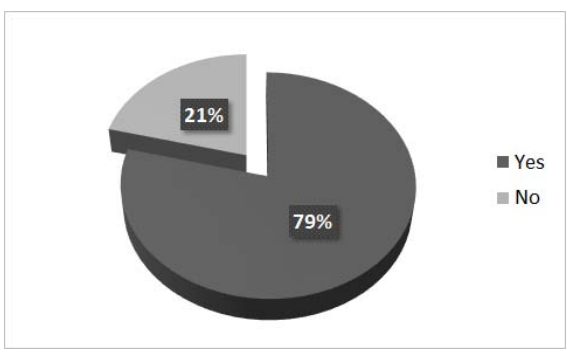

Fig. 14. Has the tool improved the student perceived knowledge on molecular phenomena? 


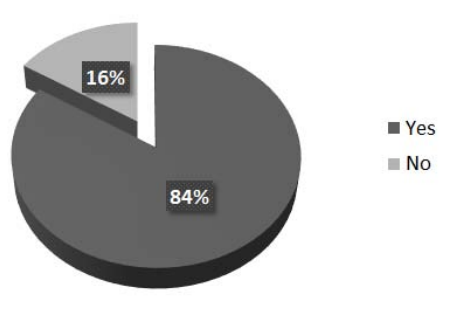

Fig. 15. Has the haptic-based tool improved your spatial abilities?

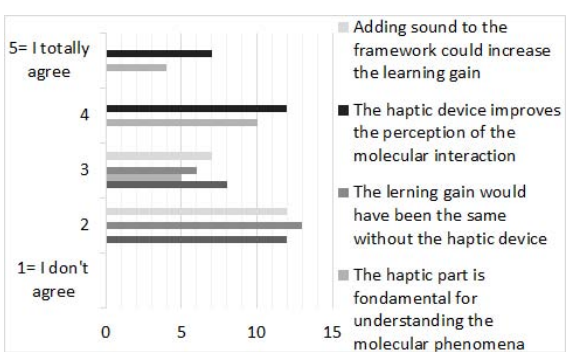

Fig. 16. Overall evaluation of the haptic-based tool

\section{Conclusions}

The present analysis helps in understanding how the application can be developed and improved for an educational purpose; the direct interaction with the users of the tool has proven to be fundamental to correctly develop the device features, especially in overcoming the difficulties that students met during the experience. In the present case, even though results are limited to a small number of participants to the test, it is generally demonstrated that the use of the haptic device as a tool for learning chemistry can help the process of understanding molecular properties and features. It is also found that there are significant improvements in the learning time. It should also be noticed that the use of a such innovative instrument enables to highly rise the interest of students in the learning process: indeed, one of the benefits is the strong increase of motivation due to the use of the haptic device. Moreover, students appear to assimilate the concepts presented through the haptic experience in an easier way. It is also noticed that, moving in a tridimentional space, student can understand the different proportions of the involved objects and how the nature and intensities of interactions can be related to spatial properties (e.g., distances from molecule).

Further works will consider the implementation of other kinds of interactions, such as covalent, ionic, and Van der Waals interactions, in order to investigate their differences and impact on the user's learning process.

\section{References}

1. Bivall Persson, P., Tibell, L.A.E.: Evaluating the Effectiveness of Haptic Visualization in Biomolecular Education: Feeling Molecular Specificity in a Docking Task. In: Proceedings of 12th IOSTE Symposium, pp. 745-752 (2006)

2. Schönborn, K.J., Bivall, P., Tibell, L.A.E.: Exploring Relationships between Students' Interaction and Learning with a Haptic Virtual Biomolecular Model. Computers and Education 57(3), 2095-2105 (2011)

3. Sourina, O., Torres, J., Wang, J.: Visual Haptic-based Biomolecular Docking. In: Proceedings of International Conference on Cyberworlds, pp. 240-247 (2008)

4. Sauer, C.M., Hastings, W.A., Okamura, A.M.: Virtual Environment for Exploring Atomic Bonding. Eurohaptics (2004) 
5. Bergamini, E., Comai, S., Corno, F., Cristini, G., Lanzani, G., Mazza, D., Metrangolo, P., Resnati, G., Terraneo, G., Brero, G., Espinoza, R., Magnanelli, E., Mahmood, K., Mazzoletti, G., Pascali, S., Rizzi, A.: A Haptic-Enhanced Framework for Chemistry Education. In: Proceedings ICCE-ECRICE, pp. 102-106 (2012)

6. Comai, S., Mazza, D.: Usability Assessment of a Multimodal Visual-Haptic Framework for Chemistry Education. In: Campos, P., Graham, N., Jorge, J., Nunes, N., Palanque, P., Winckler, M. (eds.) INTERACT 2011, Part IV. LNCS, vol. 6949, pp. 648-651. Springer, Heidelberg (2011)

7. Berg, J.M., Tymoczko, J.L., Stryer, L.: Biochemistry, 5th edn. W. H. Freeman (2002)

8. Sensable PHANTOM Omni, http://www.sensable.com/haptic-phantomomni.htm

9. Comai, S., Mazza, D.: Haptic and Visual Rendering for Multi-Modal Exploration of Molecular Information. In: BCS HCI, pp. 221-229 (2010)

10. Berman, H., Nakamura, H., Henrick, K.: The Protein Data Bank (PDB) and the Worldwide PDB. In: Encyclopedia of Genetics, Genomics, Proteomics and Bioinformatics, ch. 4(6) (2005)

11. Dupuis, M., Watts, J.D., Villar, H.O., Hurst, G.J.B.: The General Atomic and Molecular Electronic Structure System Hondo: Version 7.0. Computer Physics Communications 52(3), 415-425 (1989)

12. Leache, A.L.: Molecular Modeling: Principles and Applications, 2nd edn. Pearson (2001)

13. Stone, A.J.: Distributed Multipole Analysis, or How to Describe a Molecular Charge Distribution. Chemical Physics Letters 83(2), 233-239 (1981)

14. Kojić-Prodić, B., Molčanov, K.: The Nature of Hydrogen Bond: New Insights Into Old Theories. Acta Chim. Slov. 55, 692-708 (2008)

15. Politzer, P., Lane, P., Concha, M.C., Ma, Y., Murray, J.S.: An Overview of Halogen Bonding. Journal of Molecular Modeling 13(12), 305-311 (2007)

16. Metrangolo, P., Resnati, G.: Halogen Versus Hydrogen. Science 321(5891), 918-919 (2008) 